\title{
Towards Airborne Multipath Models for Dual Constellation and Dual Frequency GNSS
}

\author{
Michael Felux, Mihaela-Simona Circiu, Stefano Caizzone, Christoph Enneking, Friederike Fohlmeister, Markus Rippl \\ German Aerospace Center (DLR)
}

\begin{abstract}
This paper presents the initial results of an effort to develop and establish airborne multipath models for the GPS and Galileo L1/E1 and L5/E5a signals. These models will be used in dual frequency and multi constellation aeronautical navigation techniques, such as ARAIM, SBAS and GBAS. Development of the models is based on electromagnetic simulations and a large-scale data collection campaign on a variety of different airframes. In this work, results of the evaluation of the first batch of about 200 hours of flight test data with experimental equipment are shown. At a later stage, more data with avionics hardware will be collected and evaluated. This paper mainly aims at establishing the methodology and obtaining initial results as a first indication. For this purpose flight data is analyzed for periods where satellites are tracked continuously. Then, the carrier phase measurements are subtracted from the code measurements, the ionospheric divergence is removed and the bias introduced by the carrier phase ambiguities is removed. Finally, all the data is sorted, overbounded and inflated to account for the limited number of samples. The methodology also includes the removal of code errors induced by the antenna group delay, as these are suggested to be treated separately from the multipath errors.

The absolute values of the bounds for GPS L1 and Galileo E1 are larger than the existing GPS L1 model. This could be expected when being conservative at each step of the evaluation process and when using experimental hardware that is not entirely compliant with current minimum performance standards for airborne equipment. Without the conservatisms applied, the observed errors are below the Airborne Accuracy Designator (AAD A) curve. The level of residual multipath on L5/E5a is significantly smaller than that on L1/E1 and the model for the ionosphere free combination is significantly larger than the bound on L1/E1. Both these properties found in our experimental results match with theoretical expectation.
\end{abstract}

\section{INTRODUCTION}

Aeronautical navigation is increasingly based on the use of Global Navigation Satellite Systems (GNSS). It is a cornerstone of performance based navigation and enables airspace users to ensure that their navigation capabilities meet all requirements. For different phases of flight, different levels of integrity, accuracy and availability are required. The requirements for position errors reach from rather generous error budgets on the order of nautical miles horizontally with a $95 \%$ probability for the en-route phase down to 10 meters vertical with an integrity risk of just $10^{-7}$ for precision approaches. Development of techniques and augmentation systems, such as (Advanced) Receiver Autonomous Integrity Monitoring (A)RAIM and Space and Ground Based Augmentation Systems (SBAS, GBAS) continues, in order to be able to satisfy all performance requirements for current and future airspace usage.

On the side of the GNSS space segments, the European Galileo constellation continues to grow and has 17 usable satellites available and 4 more in orbit which are under commissioning at the time of writing (end of 2018). All those satellites offer signals on two frequencies (E1 and E5a) usable for aeronautical navigation. The US GPS constellation offers the L1/L5 dual-frequency capability on all satellites of the latest generation (Block IIF), of which 12 are in orbit and available for navigation. The next generation (Block IIIA) will also offer this capability and is scheduled for launch from December 2018 on.

The ARAIM concept exploits the use of dual frequency measurements to remove the ionospheric delay. The European SBAS EGNOS will provide augmentation for the Galileo and GPS constellations and for two frequencies in its V3 phase. On the GBAS side, the development of dual frequency and multi-constellation techniques to cope with challenges posed by the active ionosphere in equatorial regions is ongoing as well. All GNSS-based navigation methods need to bound any potential residual position errors. One important contribution to the positioning error is caused by multipath, i.e. signal reflections mainly on the airframe. The impact of multipath on the GPS L1 signal was characterized long time ago and appropriate error models are used for integrity 
purposes. However, no such models exist for GPS L5 and all Galileo signals. The GPS L5 signal, as well as the Galileo E5a signal use a 10 times higher chipping rate than the GPS L1 signal and are therefore less susceptible to multipath. Both signals are BPSK(10) modulated and are therefore expected to have similar performance. The Galileo E1 signal uses a BOC $(1,1)$ modulation that differs from the BPSK(1) modulation used for modulation of the GPS L1 signal. It may therefore also show different multipath characteristics. When combining the measurements on both frequencies to eliminate the first-order ionospheric delay, also the multipath contained in both measurements is combined and needs to be bounded accordingly.

Currently, the airborne multipath error models for GPS L5 and both Galileo signals are developed inside an ongoing project (Dual Frequency airborne Multipath Model for AviatioN (DUFMAN) funded by the European Commission, executed by the German Aerospace Center (DLR), Airbus Civil Aircraft, Airbus Defense and Space, Rockwell Collins and Thales Avionics) based on a combination of flight data evaluation from different aircraft, antennas and receivers as well as electromagnetic and receiver simulations. In a first step towards this model more than 200 hours of experimental flight data collected by experimental hardware on an Airbus A320 are analyzed. At later stages in the project, this data set will be supplemented by more data collected with different antennas, on a number of different airframes and with prototypes of dual-frequency and multi-constellation avionics receivers. In this paper we present the methodology of how the models are derived and the outcome of the initial phase of the project.

\section{DATA COLLECTION}

Data was collected during regular flight test operations with DLR's Airbus A320. In total, data from 78 test flights of the aircraft was collected and analyzed. These flights were mainly local flights in Europe with the exception of one transatlantic flight from Braunschweig to French Guyana and back. We analyzed a total of about 200 hours of flight data. The flights took place in the years 2015-2017. During that period data from all operational Galileo and GPS Block IIF satellites were gathered.

For the study, a modified commercial non-aviation receiver was used providing the capability to track signals on several frequencies and from different constellations. The receiver has a bandwidth of $23 \mathrm{MHz}$ and the correlator spacing was set to 0.1 chips for Galileo E1 and GPS L1 and to 1 chip for Galileo E5a and GPS L5, according to the current draft DFMC SBAS MOPS.

An avionics antenna was used that is not compliant with the recently published MOPS for dual frequency airborne antenna equipment [2]. The antenna used has a smaller axial ratio than now required. Furthermore, the group delay variations exceed the allowed limits. The group delay variations were accounted for by measuring and removing the effect from the measurements as described in the next section. The antenna was also not located in its original location but further to the back as marked by the arrow in Figure 1. That location is close to some reflectors such as the VHF antenna and may therefore be subject to increased multipath compared to the primary GNSS antennas.

The results of this initial study are therefore conservative and will be revised as more data from flight tests with representative avionics hardware becomes available during the project.

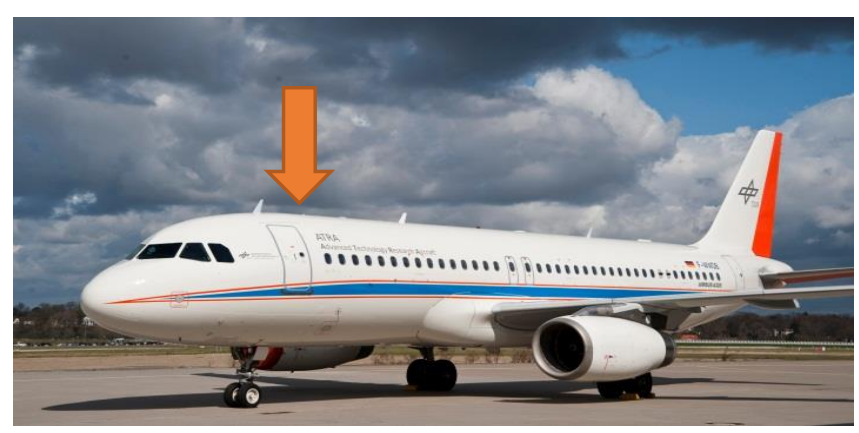

Figure 1 - The Airbus A320 research aircraft "ATRA" used for the data collection. The location of the experimental GNSS antenna is marked by the red arrow 


\section{METHODOLOGY OF DATA EVALUATION}

This section describes the individual steps undertaken to derive the actual models from the collected measurements. This methodology was established following results from previous studies such as [3]. Some steps were, however, adapted according to initial results of the study.

\section{Estimation of multipath using the dual frequency code-minus-carrier method}

In a first step, the carrier phase measurements are subtracted from the single frequency code measurements to get an estimate of the multipath contained in the data. For the purpose of this study the carrier phase is considered to be multipath free as the carrier phase multipath is on the order of millimeters and thus orders of magnitudes smaller than the code multipath. In order to account for the divergence of code and carrier phase measurements introduced by a change in the ionospheric delay over time, carrier phase measurements from a second frequency are used to estimate the divergence. For this process the combination of E1/E5a and L1/L5 was used for the respective signals.

\section{Calibration for antenna induced errors}

The errors due to antenna group delays affect the multipath estimation as they vary with angle of arrival of the signal and are thus only partially removed in the bias removal step described in the following. Since these errors are deterministic, they can be compensated for. Antenna group delays can be estimated from the antenna's gain and phase patterns [4] and a model of the GNSS receiver [5]. The antenna patterns for the antenna type installed on the aircraft were determined by measurements in an anechoic chamber. The pseudorange biases caused by the antenna were then estimated by propagation of the nominal GNSS signal through an ideal receiver with the receiver parameters (chip spacing and bandwidth) set to those of the experimental receiver.

This step is important for two reasons:

1. The errors introduced into the measurements by the antenna group delay variations were large because of the noncompliance of the antenna with the maximum limits on the group delay variation. The specified maximum variation for L1 over the pattern is about $45 \mathrm{~cm}$, whereas the variation we found with our antenna reached up to $1 \mathrm{~m}$. The calibration was describe in more detail in [6]. Therefore, it was necessary to account for them separately.

2. The errors introduced by the antenna group delay variations are dependent on the angle of arrival of the signal. Therefore, they are typically much slower changing than multipath effects and have significantly different signatures than errors introduced by multipath. For that reason characterizing those errors as statistically varying with a certain standard deviation may not be optimal.

The approach to account for the deterministic antenna group delay variations in a separate error budget also allows manufacturers or operators to obtain predictably better performance by choosing a better performing antenna.

\section{Selection of periods of continuous tracking and removal of the integer ambiguities}

In the next step the data is screened for cycle slips in order to obtain periods of uninterrupted tracking of the signals. Over these continuous segments of data the bias due to the carrier phase ambiguities (resulting from subtracting the ambiguous carrier phase measurements from the unambiguous code measurements) is removed. In previous studies only the mean value over a continuous period of tracking was removed. That method was sub-optimal in that residual biases may remain, e.g. when very short datasets including multipath are considered. In the present study, the bias is estimated by evaluating the median over portions of data prescreened to ensure low multipath exposition. Details of this method were described in [6].

\section{Code carrier smoothing}

As the models have to bound the residual errors in the pseudoranges after code-carrier smoothing is applied, the same code-carrier smoothing is applied to the obtained data. Here, the same smoothing time constant as in the positioning algorithms needs to be used in order to obtain representative results. For this study and the results presented here, a smoothing time constant of 100 seconds was used. It is, however, possible to also evaluate different smoothing time constants, e.g. in case a shorter longer smoothing time constant is used (e.g. similar to the 30 seconds in the GBAS approach service type D or longer smoothing time constants for improved multipath reduction). 


\section{Elevation binning and determination of standard deviation}

Data from all satellites is then sorted into bins of satellite elevation. The satellite elevation is computed in two ways: once as elevation of the satellite above the horizon and once as elevation of the satellite with respect to the aircraft attitude. The second option was considered additionally, as multipath is mostly resulting from reflections caused by the airframe. Both methods led to similar results with our installation. However, due to a non-ideal antenna location on the aircraft the measurements were affected by multipath from different reflectors. Furthermore, the multipath rejection capability of the antenna is not very good, so generally high levels of multipath were experienced for all angles of arrival. This behavior is subject to further investigations with data from the primary antennas on different aircraft.

The mean of the data in each elevation bin is close to zero for all datasets processed. The standard deviation of the data was then determined from all independent samples, where the rate of independent samples is twice the smoothing time constant, i.e. every 200s. The results (after calibration for the antenna group delays) are shown in Figure 2. In this example the bin size was chosen to be $2^{\circ}$ and constant for all elevations. For the future, the bin size may also be adapted, depending on the number of samples per bin (in order to not obtain very different results just by accounting for a limited number of samples per bin as described later).

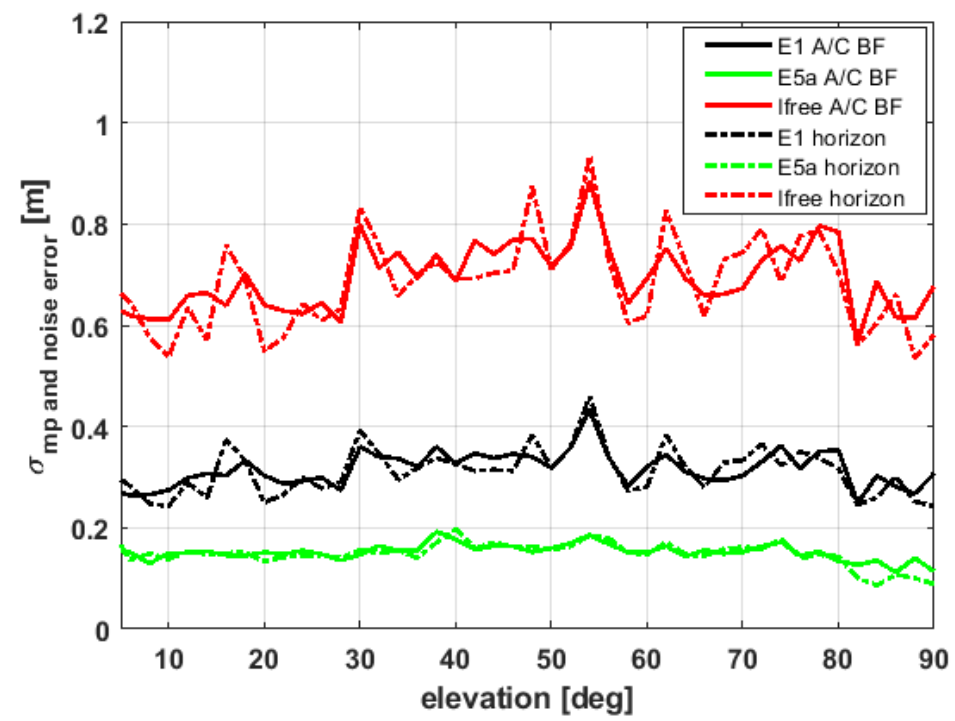

Figure 2 Standard deviation of the estimated multipath from measurements for Galileo E1 (black), E5a(green) and Ifree combination of the two (red). The solid line shows the results for satellite elevation w.r.t. the aircraft body frame, the dashed lines w.r.t. horizon.

\section{Overbounding}

Generally, the multipath per bin is then described by a zero-mean Gaussian distribution. As it was observed in the data, the distribution of the multipath estimates in each bin is, however, not properly described by a Gaussian distribution. In all bins it was found that the weight of the tails was heavier than that of the fitted normal distribution. This would mean that the probability of having large multipath induced errors is larger than suggested by the Gaussian fit. Therefore, an overbounding is suggested to ensure that the risk of larger multipath induced errors is not underestimated. The overbounding process is illustrated in Figure 3 Shown in black is the cumulative distribution function (CDF) and 1-CDF of the sample data for Galileo E1 in one bin (here the elevation from $24-26^{\circ}$ ). The blue curve is a Gaussian fit to the sample data. It can be observed that in the range of about $\pm 0.5 \mathrm{~m}$ the Gaussian fit is quite well in accordance with the sample data. However, for larger errors the Gaussian fit model underestimates the probability of occurrence by orders of magnitude. The green curve then shows a Gaussian overbound to ensure that the tails of the distribution are properly bounded and the risk of having errors larger than suggested by the Gaussian model is mitigated. 


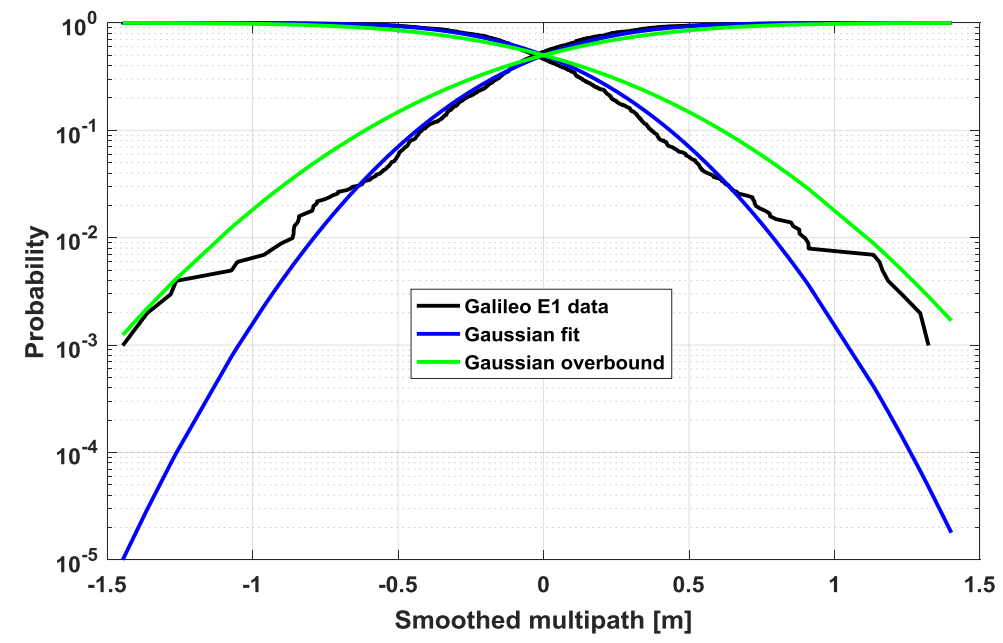

Figure $3 \mathrm{CDF}$ and 1-CDF of sample data of Galileo E1 in the elevation bin 24-26 (black), Gaussian fit to the data (blue), Gaussian overbound (green)

\section{Inflation to account for limited amount of data}

Finally, the models were derived based on a limited number of samples. In the relevant integrity models, the integrity risk is on the order of $10^{-7}$. An inflation factor in order to ensure statistical representability of the results is determined based on the Chi-Squared distribution of the standard deviation. The number of samples used to determine the model and the resulting inflation factor for a 95\% confidence is shown at the example of Galileo data in Figure 4. The choice of bin size, and therefore the number of samples per bin and the resulting inflation factor of course plays a role in determining the models. For the final version it is expected that the amount of data available will be large enough that no significant inflation is going to be necessary. A different binning, especially for higher elevations, may be chosen anyways in the future in order to obtain similar inflation factors for all elevations and reduce the effect this step has on the overall results.
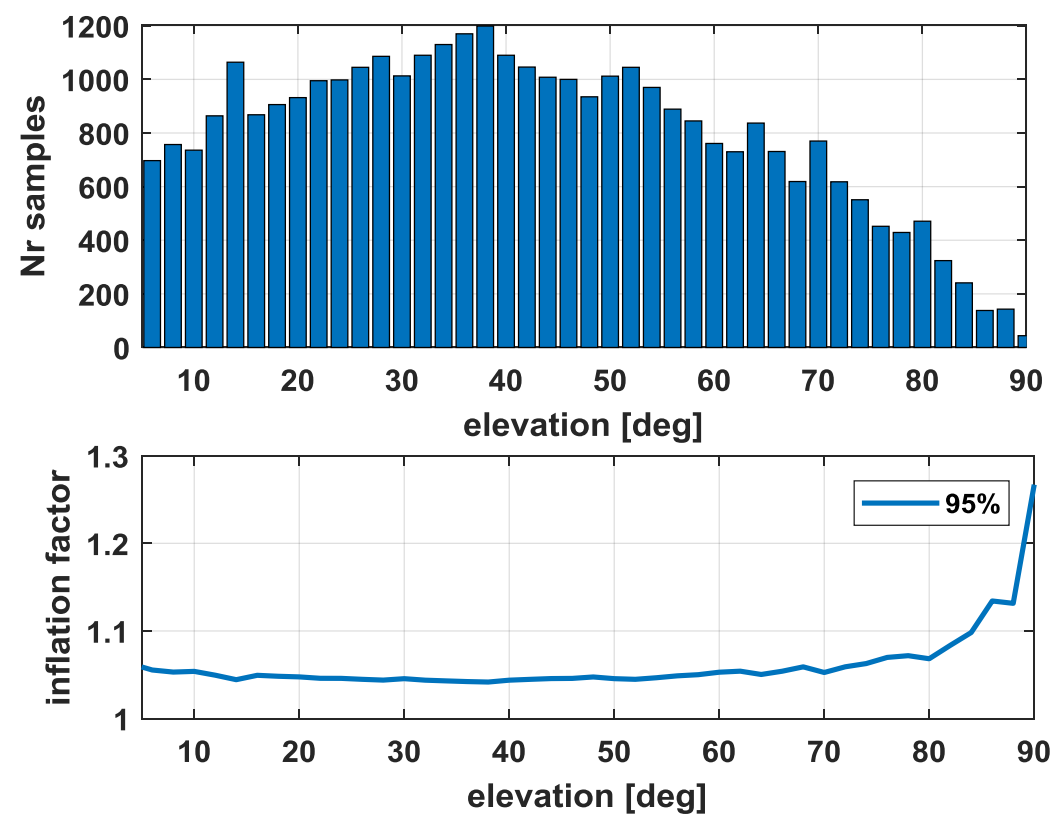

Figure 4: Number of samples per bin and resulting inflation factor to account for limited number of samples for Galileo 


\section{RESULTS AND DISCUSSION}

Based on the preliminary set of data, the following initial results for airborne multipath were derived and are shown in Figure 5 for Galileo and Figure 6 for GPS. The green curves show the results for E5a/L5, the black curves for E1/L1 and the red curves for the ionosphere free combination of E1/E5a and L1/L5, respectively. It should be noted again here that these curves will change as data from representative hardware and installations becomes available. The goal of the work so far was mainly to establish the methodology and tools and get first insights on the approximate performance levels.

The results shown are more conservative than the existing sigma_air models. The main reasons for this result are:

1. Use of experimental and non-MOPS compliant hardware, as well as antenna installation in non-optimal location on the fuselage

2. More conservative statistical approach in terms of

a) overbounding to account for non-Gaussian distribution of samples in each bin

b) inflation to account for limited number of independent samples in each bin.

At this stage of the project, it is yet to be decided whether all those inflation steps are required to standardize the resulting model. The final performance levels will be determined based on a large amount of flight test data from representative installations on different airframes which may leverage some of the conservatism.

In contrast to the existing model, no significant elevation dependency was found. This outcome may, however, result from the specific installation and may change with data from representative installations and the use of different antennas.

It should be noted again that the effect of antenna group delay variations was excluded from the model and therefore needs to be accounted for separately in integrity considerations. This separation of the two error components was necessary in the process of data evaluation but is also suggested in the operational context as the behavior of the multipath error and antenna induced error is significantly different.

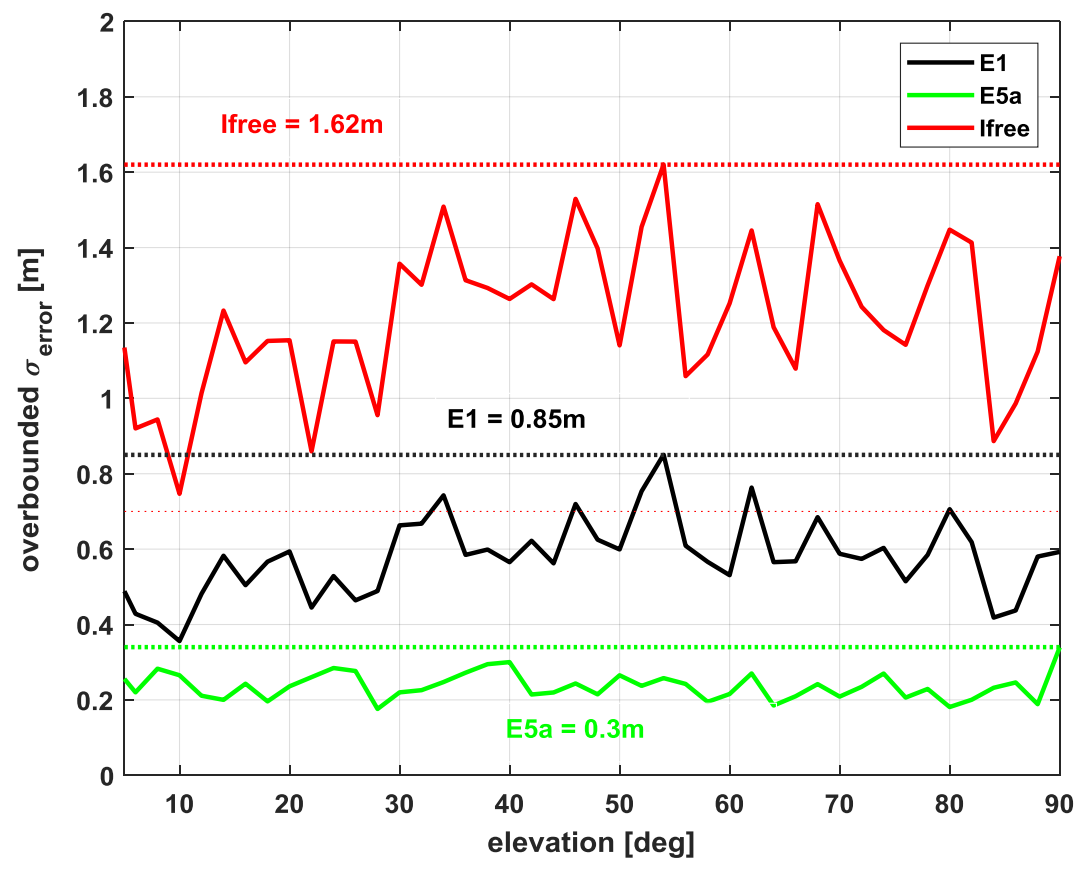

Figure 5: Preliminary results for Galileo 


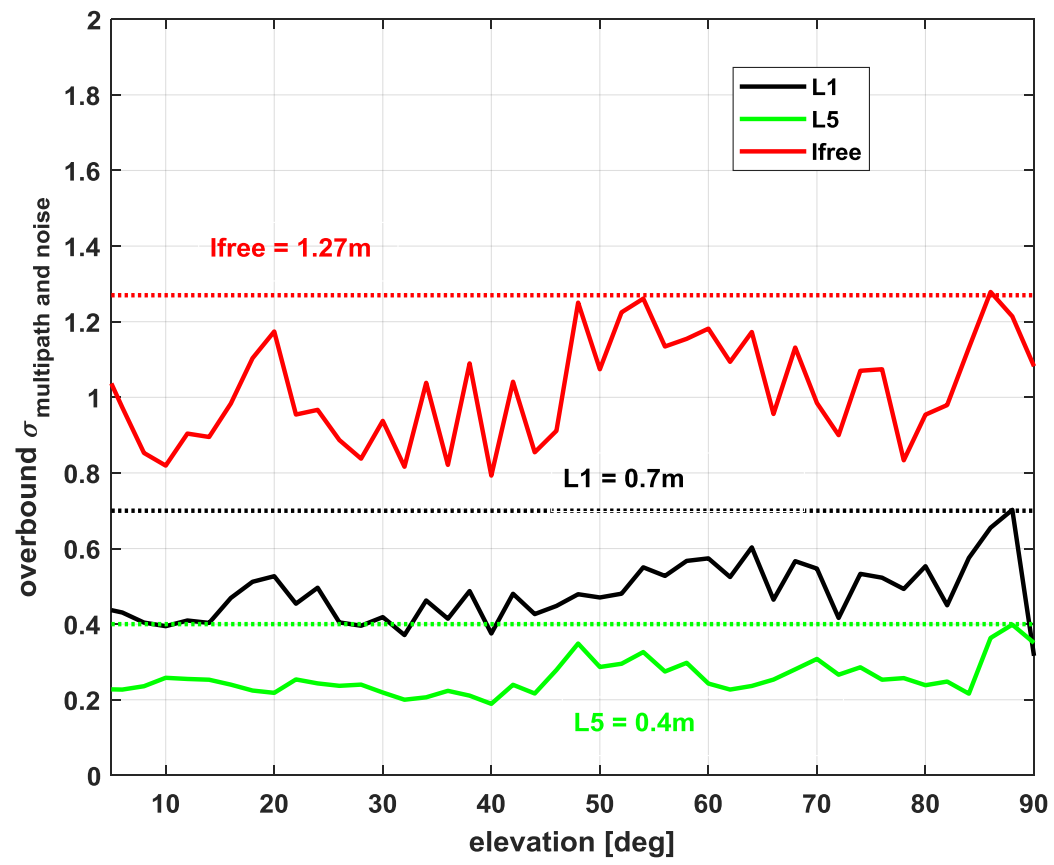

Figure 6: Preliminary results for GPS

\section{ACKNOWLEDGMENTS}

The work presented in this paper was carried out in the frame of the project DUFMAN funded by the European Commission (DGGROW / JRC). Opinions expressed herein are those of the contractor only and do not represent the contracting authorities' official position.

\section{REFERENCES}

References should be numbered consecutively in the text with numbers in brackets, and appear at the end of the paper in the format shown below:

[1] "Concept of Operations (CONOPS) for Dual-Frequency Multi-Constellation (DFMC) Global Navigation Satellite System (GNSS)", ICAO Navigation Systems Panel, April 2018

[2] DO-373 - Minimum Operational Performance Standards for GNSS Airborne Active Antenna Equipment for the L1/E1 and L5/E5a Frequency Bands, RTCA 2018

[3] Murphy, T., Harris, M., "Multipath Flight Test Program \& Results”, Boeing report D6-83447-3 (2005)

[4] Caizzone, S., Circiu M.-S., Elmarissi W., Enneking C., Felux M., Yinusa K., "Antenna Influence on GNSS Pseudorange Performance for Future Aeronautics Multi-frequency Standardization ”, NAVIGATION, in press (2018)

[5] Vergara M., Sgammini M., Thoelert S., Enneking C., Zhu Y. and Antreich F., "Tracking Error Modelling in Presence of Satellite Imperfections", NAVIGATION, 63: 3-13. doi: 10.1002/navi.129.

[6] Circiu M.-S., Caizzone S., Felux M., Enneking C. and Meurer M. "Improved Airborne Multipath Modelling", Proceedings of $31^{\text {st }}$ International Meeting of the Satellite Division of The Institute of Navigation (ION GNSS+ 2018), Miami, FL, USA, September 2018, pp. 130-138. 